\title{
CORRECTIONS
}

\section{Interpreting asymptomatic bacteriuria}

An editorial oversight while finalising the proofs of this Practice article by Martin Cormican and colleagues (BMJ

2011;343:d4780, doi:10.1136/bmj.d4780) led to the omission of the important prefix "non." In the second paragraph of the

"Avoidance of testing" section the fourth sentence should have started: "Treatment of asymptomatic bacteriuria is not recommended in non-pregnant women [not "pregnant women"] of any age."

Cite this as: $B M J 2011 ; 343: d 5084$ 